\title{
Depot-specific response of adipose tissue to diet-induced inflammation: the retinoid-related orphan receptor $\alpha$ involved?
}

\author{
Sarah Kadiri ${ }^{1,2}$, Martine Auclair ${ }^{1,2}$, Jacqueline Capeau ${ }^{1,2}$ and Bénédicte Antoine ${ }^{1,2,3}$
}

1 Inserm U938, Paris F75012, France

2 UPMC Université Paris 06, UMR_S 938, Paris, F-75012, France

3 CNRS, UMR_S 938, Paris, F-75012, France

Corresponding author: B. Antoine

Address for correspondence:

Faculté de Médecine site Saint-Antoine, 27 Rue Chaligny, 75012 Paris, France

tel 331400113 51, fax 331400114 32,

benedicte.antoine@inserm.fr

Running title: ROR $\alpha$ involved in fat depot-specific inflammation

Keywords: Fat depots, inflammation, ROR $\alpha$, western diet, insulin sensitivity

Word Count: 3500

Funding: SK was the recipient of PhD grants from the Ministère de l'Education Nationale et de la Recherche and from CODDIM. BA is a CNRS researcher. 


\section{What is already known about this subject?}

The increased mass of visceral fat depot is associated with metabolic risk, while the expansion of subcutaneous fat mass appears neutral or even protective regarding the metabolic risk, both in humans and rodents. Recent studies performed to understand this depot-specific behaviour of adipose tissue revealed differences in developmental lineage of adipocyte as well as in their micro-environment.

With regard to the metabolic risks, most studies have focused primarily on the visceral depot, while only few publications dealt with the elucidation of how increased amounts of subcutaneous depot could be neutral or even beneficial regarding the metabolic effects. Understanding the underlying mechanisms for these differences is of importance in the course of preventing the ongoing obesity and diabetes epidemic.

The orphan nuclear factor ROR $\alpha$ is involved in several processes that are dysregulated in expanding adipose tissue, such as inflammation, adipogenesis, circadian rhythms and lipid homeostasis. Its role of in the inflammation of adipose tissue thus remains controversial.

\section{What does this study add?}

ROR $\alpha$ expression impacts the basal inflammation status of adipose tissue: when ROR $\alpha$ is inefficient, the level of basal inflammation decreases. Such data confirm a "pro-inflammatory" role of ROR $\alpha$ in physiological conditions, very likely because it was shown as a positive actor in the basal transcription of several cytokines.

All along the diet-induced fat expansion in wild-type mice, the inflammation of visceral fat increases (as generally observed in mice and human). Our data show here that, whenever the subcutaneous fat also hypertrophies, its inflammation level decreases. This differential behavior of the fat depots is associated with inverse effect on Insulin sensitivity, thus impacts on the functionality of the fat depot.

We then show that ROR $\alpha$ is regulated in obesity in a depot-specific manner and has depot specific function. In response to a western diet (WD), ROR $\alpha$ and its target-gene I? $\mathrm{B} \alpha$, increases in the stroma vascular fraction (SVF) in the non-adipocyte fraction of subcutaneous fat and not of visceral fat. These findings suggest an anti-inflammatory role for ROR $\alpha$ in response to WD, which occurs at the level of stroma vascular fraction of subcutaneous fat, thus possibly participating to the "healthy" expansion of subcutaneous fat. 


\section{Summary}

Objective: Epididymal adipose tissue (EAT), a visceral fat depot, is more closely associated with metabolic dysfunction than inguinal adipose tissue (IAT), a subcutaneous depot. We evaluated whether the nuclear receptor ROR $\alpha$, which controls inflammatory processes, could be implicated.

Methods: We compared EAT and IAT in a ROR $\alpha$ loss-of-function mouse (sg/sg) and in wild-type (WT) littermates, fed a standard diet (SD) or a western diet (WD), to evaluate how ROR $\alpha$ expression impact on inflammatory status and on insulin sensitivity (IS) of each fat depot according to the diet.

Results: Sg/sg mice fed the SD exhibited a decreased inflammatory status and a higher IS in their fat depots than WT mice. WD-induced obesity has distinct effects on the two fat depots. In WT mice, EAT exhibited increased inflammation and insulin resistance while IAT showed reduced inflammation and improved IS, together with a depot-specific increase of $R O R \alpha$, and its target-gene $I \kappa B \alpha$, in the stroma vascular fraction (SVF). Conversely, in sg/sg mice, WD increased inflammation and lowered IS of IAT, but not EAT.

Conclusions: Our findings suggest an anti-inflammatory role for ROR $\alpha$ in response to WD, which occurs at the level of SVF of IAT, thus possibly participating to the "healthy" expansion of IAT. 


\section{Introduction}

Obesity is characterised by hyperplasic and hypertrophic adipose tissue expansion, which is associated with a chronic low-grade inflammation. An emerging body of research suggests that inflammatory processes that occur within the intra-abdominal adipose tissue depot of humans and rodents are causatively related to many of the symptoms of the metabolic syndrome (1). This remodelling of adipose tissue inflammatory status is due to infiltration by several immune cell types including macrophages (2), T lymphocytes, natural killer cells and neutrophils (3). The inflammatory adipose tissue releases pro-inflammatory cytokines, such as IL-6, TNF $\alpha$, IL1 $\beta$, which impair insulin sensitivity (4).

However, these modifications particularly concern the visceral fat depot, whose increased mass is associated with metabolic risk, while increased subcutaneous fat mass appears neutral or even protective regarding the metabolic risk, both in humans and rodents $(5,6)$. Recent studies performed to understand this depot-specific behaviour of adipose tissue revealed differences in developmental lineage of adipocyte precursors $(7,8)$ as well as in their microenvironment $(9)$. This implies that the immune cells, besides adipocytes and adipocyte precursors, could also differ according to the fat depot location. With regard to the metabolic risks, most studies have focused primarily on the visceral depot, while only few publications dealt with the elucidation of how increased amounts of subcutaneous depot could be neutral or even beneficial regarding the metabolic effects.

Members of the nuclear hormone receptor family are abundantly expressed in tissues with major metabolic activity, such as adipose tissue, liver and skeletal muscle. In vivo studies have revealed that some of the orphan nuclear receptors regulate the pathophysiology of obesity and insulin resistance (for a review see ref 10). The orphan nuclear factor ROR $\alpha$, for example, is involved in several processes that are deregulated in expanding adipose tissue, such as inflammation, adipogenesis, circadian rhythms 
and lipid homeostasis (see ref. (11) for a review). A mouse ROR $\alpha$ loss-of-function mutant - the staggerer (sg/sg) mice - is protected against high fat diet-induced obesity (12) and related systemic insulin resistance (13). The sg/sg mice have a global $\operatorname{ROR} \alpha$ defect that results in decreased and dysfunctional expression of the two mouse $\operatorname{ROR} \alpha$ isoforms, $\operatorname{ROR} \alpha 1$ and $\operatorname{ROR} \alpha 4$. The latter form is predominantly expressed in adipose tissue. These mice are lean and their pre-adipocytes better differentiate to adipocytes in vitro as compared to their wild-type (WT) mice counterparts $(14,15)$. We recently demonstrated that glyceroneogenesis, a pathway that controls fatty acids homeostasis, is a new target of ROR $\alpha$, thus limiting the storage of lipids into the adipocytes from $\mathrm{sg} / \mathrm{sg}$ mice (16).

When fed a high-fat (HF) diet, sg/sg mice express a lower level of immune and inflammatory genes in their epididymal fat than WT mice on the same diet (13); a pro-inflammatory role for ROR $\alpha$ was presumed in high fat diet-induced inflammation of visceral adipose tissue by these authors. To the contrary, others studies suggested that ROR $\alpha$ may have a protective role during acute inflammation of muscle cells and macrophages (17-20). The role of ROR $\alpha$ in inflammation thus remains controversial.

Finally, sg/sg mice exhibited increased levels of insulin-stimulated phosphorylated Akt and of glucose uptake in muscle (21), but the insulin sensitivity (IS) of their adipose tissues has not yet been investigated.

The purpose of the present work was to decipher the role of ROR $\alpha$ in adipose tissue inflammation and IS under physiological condition, and in a context of excessive caloric intake. Here, we compared sg/sg mice with their WT littermates, first when fed a standard diet (SD), and after highcarbohydrate and high-fat western diet (WD). Only such an approach allowed a comparison of the diet related-effect on each genotype. We systematically analyzed both visceral (epididymal) and subcutaneous (inguinal) adipose tissues (EAT and IAT) because of their different impact on metabolism. 


\section{Material and methods}

Animals and tissues. Dr J-L Danan gave us the heterozygous sg/+ mice, which came from $\mathrm{Dr} J$ Mariani's laboratory. Homozygous sg/sg mice were obtained by crossing heterozygous mice. All experimental procedures on mice were conducted in accordance with the guidelines of the Charles Darwin Ethics Committee (Ce5/2010/034). Three-month-old male mice were fed ad libitum either with a high-fat, high-carbohydrate 'western' diet (WD) (40\% kcal fat and 40\% kcal carbohydrates, TD96132, Harlan) or a standard diet (SD) for 12 weeks. After $4 \mathrm{~h}$ fasting, mice were euthanized by cervical dislocation and adipose tissues removed.

Immunohistochemistry. Adipose tissue samples were fixed in formol and embedded in paraffin. Sections were then immunostained for CD68 (MCA1957, AbD Serotec). For each mouse, 300-400 adipocytes/macrophages were counted in each fat depot.

RNA extraction, cDNA synthesis and quantitative PCR. Adipose tissue mRNA was extracted by using the RNeasy Lipid Tissue MiniKit (Qiagen), then reverse-transcribed by using the High-Capacity cDNA Reverse Transcription Kit (Applied Biosystems). PCR of the genes of interest was performed using a Light Cycler 480 Real-Time PCR System (Roche) and the specific primers listed as followed:

F4/80: 5'-GCAAGGAGGACAGAGTTTATCGTG-3',5'-CTTTGGCTATGGGCTTCCAGTC-3';

CD68:5'-CCAATTCAGGgTGGAAGAAA-3',5'-CTCGGGCTCTGATGTAGGTC-3';

IL-6: 5'- AGAAGGAGTGGCTAAGGACCAA -3', 5'-ACGCACTAGGTTTGCCGAGTA-3'

TNF $\alpha$ : 5'-TCTTTCTCGAACCCCGAGTGA-3', 5'-CCTCTGATGGCACCACCAG-3'

IL-1 $\beta$ : 5'-ACACTCCTTAGTCCTCGGCCA-3', 5'-CCATCAGAGGCAAGGAGGAA-3'

IL-10: 5'-ACGGCGCTGTCATCGATT-3', 5'-GGCATTCTTCACCTGCTCCA-3'

PAI-1: 5'-CTATGCTGACATGACCACAGC-3', 5'-CTGAGCCATCATGGGCACAGAG-3'

ROR $\alpha:$ 5'-CGTTTGGCAAACTCCACC-3',5'-GATTGAAAGCTGACTCGTTCC -3' 
Expression of each gene was quantified by cycle threshold values and was normalized to HPRT (hypoxanthine phosphoribosyltransferase) mRNA.

Functional analysis in explants. Adipose tissue was minced into very small pieces (5-10 mg) and incubated overnight in DMEM containing $5 \mathrm{mM}$ glucose and $0.5 \%$ bovine serum albumin (BSA), then incubated (or not) with $100 \mathrm{nM}$ insulin in DMEM containing $17 \mathrm{mM}$ glucose for 10 min before western blot analysis or 2-deoxy-D-glucose uptake assay (21) to evaluate insulin sensitivity. The 48 h-culture media were used to measure cytokine secretion (MCP-1/CCL-2, TNF- $\alpha$, IL-10) by ELISA (Prepotech).

Protein extraction and western blot analysis. Adipose tissue was extracted using Tissue Lyzer (Qiagen). Extracts were centrifuged first at $10,000 \mathrm{rpm}$ and $4^{\circ} \mathrm{C}$ for $8 \mathrm{~min}$ to remove the fat coat, and then at $14,000 \mathrm{rpm}$ and $4^{\circ} \mathrm{C}$ for $10 \mathrm{~min}$ to remove insoluble material. Supernatants were subjected to SDS-PAGE and western blotted with antibodies against pAkT (sc-7985), AkT (sc-8312), ROR $\alpha$ (sc-

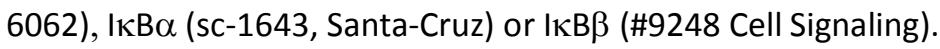

Adipose tissue sub-fractionation: Fat pads were excised and adipocytes were isolated from the SVF after collagenase digestion (Liberase TM, Roche) as previously described (22).

Statistical analysis. The data are presented as means +/- SD. Statistical analysis was performed by one-way or two-way ANOVA or by unpaired Student T test; $p<0,05$ was considered significant. 


\section{Results}

Metabolic status and adipose tissue expansion according to the genotype and in response to WD

When fed the SD, the total mass of white adipose tissue in the sg/sg mice was $50 \%$ less than in the WT controls. Parallely, their mean adipocyte surface area in IAT and EAT sections was four-fold smaller than that in WT controls (Fig S1), as previously observed (23).

When fed the WD, WT and sg/sg mice gained $5 \mathrm{~g}$ and $2 \mathrm{~g}$ respectively. This was associated with a relative increase in the white adipose tissue mass of about four-fold in WT mice and two-fold in sg/sg mice, with the same impact on each fat depot. The mean adipocyte area in EAT and IAT from WT and sg/sg mice increased two- and four-fold respectively (Fig S1). WT mice became glucose intolerant and insulin-resistant whereas sg/sg mice were protected against the insulin-resistance induced by our "high-carbohydrate and high-fat" WD (not shown), as also described in response to a $60 \%$ high fat diet (13).

The inflammatory profiles of epididymal and inguinal fat depots differ according to the genotype and in response to WD

To examine the inflammatory status of IAT and EAT in WT and sg/sg mice fed the SD or WD, we measured the mRNA levels of two macrophages markers (F4/80 and CD68), four proinflammatory cytokines (IL6, TNF $\alpha$, IL1 $\beta$, PAI-1) and one anti-inflammatory cytokine (IL10) (Fig 1). We evaluated the number of CD68+ cells by immunohistochemistry. We also measured the secretion of the chemokine MCP-1/CCL2 and of two cytokines (TNF $\alpha$, IL10) by ELISA (Fig 2).

\section{- $\quad$ Compared basal inflammatory status of adipose fat depots in WT and sg/sg mice fed the SD.}

When compared to their corresponding WT depots, EAT and IAT of sg/sg mice fed the SD expressed lower amounts of most of the tested markers, when regarding the mRNA level (Fig 1) or the chemokine/cytokine secretion (Fig 2). When EAT and IAT were compared in WT mice fed the SD, we observed that the inflammatory status of IAT was globally higher than that of EAT (IAT had higher 
number of CD68+ macrophages and secretion of CCL2 and expressed higher mRNA levels of TNF $\alpha$, IL1 $\beta$ and IL10 than the EAT) (Fig S2A). By contrast, in sg/sg mice fed the SD, the inflammatory status of IAT was not higher than that of EAT (lower mRNA expression of IL1 $\beta$ and PAI-1) (Fig S2A). Taken together, our data show for the first time that the absence of ROR $\alpha$ has an impact on the basal inflammation status of fat depots, and particularly in IAT.

- Different inflammatory profiles in fat depots according to WD in the two mouse genotypes

As expected, WD increased the inflammation of EAT in WT mice as compared to SD : higher amount of F4/80, IL6, IL10 and PAI-1 mRNAs (Fig 1A') and about a two-fold increase of the number of CD68+ macrophages (Fig 2A) and of CCL2 secretion (Fig 2B) together with increased secretion of TNF $\alpha$ and IL10 (not shown). However, in IAT, the expression of the majority of inflammatory markers was decreased by WD (Fig 1 $B^{\prime}$ ), as well as the number of CD68+ macrophages (Fig 2A) and the amount of secreted CCL2 (Fig2B).

By contrast, in sg/sg mice, WD increased the inflammatory status of IAT (Fig 1 $\mathrm{B}^{\prime}$ ), but not of EAT (Fig $1 A^{\prime}$ ). These data were confirmed by the observation of an increased secretion of CCL2 (Fig2B) and of TNF $\alpha$ and IL10 (Fig 2C) by IAT from sg/sg mice fed the WD vs fed the SD.

These data show that, in WT mice fed the WD, the inflammatory markers increased in EAT while they decreased or plateaued in IAT, whereas the opposite occured in sg/sg mice fed the WD.

Insulin sensitivity of epididymal and inguinal fat depots differs according to genotype and to diet.

To investigate whether these differing inflammatory profiles of adipose tissues in WT and $\mathrm{sg} / \mathrm{sg}$ mice correlated with differences in insulin sensitivity, we evaluated the ability of insulin to activate the insulin receptor signaling enzyme Akt. We measured the level of activated P-Akt in the absence or the presence of insulin, relative to the total Akt levels, in the two tissue types of both mouse strains fed either the SD or the WD. In sg/sg mice, the insulin sensitivity of both tissues was better than in WT mice and, in both strains fed the SD, we observed a higher level of P-Akt in EAT 
than in IAT (as illustrated by Fig. 3A), indicating that EAT was more sensitive to insulin than the IAT. We further assessed these observations by analyzing 2-DOG incorporation in response to insulin as shown in Fig 3B.

When WT mice were fed the WD, the level of insulin-stimulated P-Akt in EAT was lower than in the WT mice on the SD, indicating a diet-increased insulin resistance in EAT (Fig 3C), whereas in IAT, the response to insulin was improved by the WD regimen (Fig 3D). In the sg/sg mice on the WD, by contrast, insulin sensitivity was maintained in the EAT but decreased in the IAT (see histograms of Fig $3 \mathrm{C}$ and $\mathrm{D})$. Thus, there is a direct correlation between insulin resistance and inflammation in the two strains in response to WD. However, it was EAT that became insulin resistant in the WT mice, whereas it was the IAT that exhibited decreased insulin sensitivity in $\mathrm{sg} / \mathrm{sg}$ mice in response to the WD.

\section{ROR $\alpha$ protein increased in IAT of WT mice fed the WD as well as IKB protein.}

WD resulted in reduced inflammation in IAT of WT mice, this response being lost in IAT of $\mathrm{sg} / \mathrm{sg}$ mice. We therefore suspected a role for ROR $\alpha$ in these differences. To this end, we measured ROR $\alpha$ expression in EAT and IAT in WT mice as well as in sg/sg mice maintained on either regimen (Fig. 4A). In SD-fed sg/sg mice, ROR $\alpha$ mRNA levels were lower than in WT mice, as expected (12), and they did not change in response to the WD. In SD-fed WT mice, ROR $\alpha$ expression was similar in the two fat depots. However, because ROR $\alpha$ is a member of the clock "machinery", we checked the circadian variation of the ROR $\alpha$ protein. We observed a higher amount of the protein in EAT than in IAT of WT mice fed the SD, with a peak during the dark period (Fig 4B). When WT mice were fed the WD, ROR $\alpha$ expression did not change in the EAT but, by contrast, markedly increased in the IAT when compared to the same tissue in SD-fed WT mice (Fig 4A). This was confirmed by checking the amount of ROR $\alpha$ protein that increased in IAT of WT mice in response to WD (Fig 4C).

I $\mathrm{B} \alpha$ protein, an inhibitor of NF-KB-mediated transcriptional activation, being shown to be a target gene of ROR $\alpha$ (17), we first checked if this WD-induced amount of ROR $\alpha$ in the SVF of IAT was 
associated with a depot-specific increase of the IKB family. Our data indeed show that WD increased IKB $\beta$ protein in the IAT of WT mice (in parallel with the increase of ROR $\alpha$ ) and not, in EAT (Fig 4D).

To reveal where this WD-increased amount of ROR $\alpha$ protein took place, we separated adipocytes from the SVF of both fat depots from WT mice fed the SD or the WD. Our data show that both the adipocytes and the SVF expressed ROR $\alpha$ protein (Fig 5A). However, in response to WD, there was only an increase of ROR $\alpha$ protein in the SVF of IAT, and not of EAT, whereas its amount decreased in adipocytes from both fat depots. Thus, our data show that there is a profound difference in the response of EAT and IAT to the WD, in terms of induction of ROR $\alpha$ expression that only occurs in the SVF of IAT. This suggests a WD-induced response of the non-adipocyte fraction, and not of the adipocytes, that is depot-specific of IAT.

Finally, we confirmed that this WD-induced amount of ROR $\alpha$ in the SVF of IAT was associated with a depot-specific increase of $I \kappa B \alpha$ (Fig 5B). This confirms a depot-specific role for ROR $\alpha$ that could contribute to the protection of diet-induced inflammation by providing an increased amount of an NF-KB inhibitor in the non-adipocyte fraction of IAT.

\section{Discussion}

Our investigation offers insights into a new mechanism underlying for the differences in dietinduced expansion of visceral and subcutaneous fat depots and their metabolic consequences. While expansion of the EAT results in its increased inflammation and insulin resistance, as generally observed, our data highlight that expansion of the IAT remarkably differs because it is not only metabolically neutral but associated with decreased inflammation and increased insulin sensitivity. Elucidating the basis of such an "healthy" expansion of subcutaneous fat is of importance with regards to the prevention of diet-induced metabolic syndrome. In this study, we discover a depotspecific induction of ROR $\alpha$ in the IAT of WT mice fed the WD that is associated with an increase of IKB protein, an inhibitor of NFKB-mediated transcriptional activation. Our data suggest that ROR $\alpha$ 
could act as a negative regulator of the diet-induced inflammatory response in the subcutaneous fat depot, where it is expressed by non-adipocyte cells, perhaps contributing to the relative protection of IAT in diet-induced metabolic syndrome.

To mimic the cafeteria-type diet of humans, we choose to feed WT and sg/sg mice a diet that contains equal amounts of carbohydrates and lipids (40\%) that we called "western" diet, rather than the often-used HF diet (60\% of lipids and very few carbohydrates) that is not representative of any common "human" diet. Twelve weeks of this WD had the expected metabolic consequences for WT mice : increased insulin resistance at the systemic level and fat weight gain. Accordingly, in EAT, the inflammatory response was enhanced and the insulin sensitivity decreased compared to animals on the SD. Surprisingly, we observed that the IAT of WT mice responded inversely to WD than their EAT : the inflammatory status declined and insulin sensitivity increased.

Several reports already mentioned that IAT has a lower inflammatory status than EAT in mice having been fed a HF diet (24-26); however, a reduced level of inflammation as compared to SD-fed mice was unexpected. For instance, when fat expansion was analyzed all along the HF diet (26), EAT was shown to initiate adipocyte death sooner than IAT; such adipocyte death in EAT being timely linked to the recruitment and /or phenotypic switch of macrophages, AT inflammation and wholebody insulin resistance. However, none of these events were detectable in IAT before 12 weeks of HF diet (26). Our data confirm similar behavior - a distinct impact of WD diet on EAT and IAT inflammation whereas similarly hypertrophied, but they also bring the new notion that IAT can protect itself against the WD-induced inflammation. This new observation was perhaps allowed by the use, in our study, of a more balanced diet (WD instead of a HF diet).

This WD-induced decrease of inflammation in IAT was detectable because of the higher level of basal inflammatory status of IAT than EAT in WT mice fed the SD (as illustrated in Fig S2).

Accordingly, several investigations performed in mice and rats on chow diet have noticed a higher basal inflammatory status in IAT than in EAT (27-29) as well as a higher content of cells not related to the adipocyte lineage in IAT (30). For instance, mast cells, that can act as negative as well 
as positive regulators of immunity (31), are more prevalent in subcutaneous than visceral fat from lean mice (25) as well as from humans (32). Our observation of higher amounts of ROR $\alpha$ and IKB $\alpha$ and $\beta$ protein in EAT than in IAT of SD-fed WT mice could alternatively participate to the lower basal inflammation status of EAT than IAT.

The next addressed question was how IAT actively protects itself against diet-induced inflammation and IR. The observation of an opposite phenomenon in the IAT of sg/sg mice fed the WD suggested us a role for ROR $\alpha$ as a possible actor of an anti-inflammatory process in the IAT of WT mice. Accordingly, we found a depot-specific increase of ROR $\alpha$ in the IAT of WT mice fed the WD,

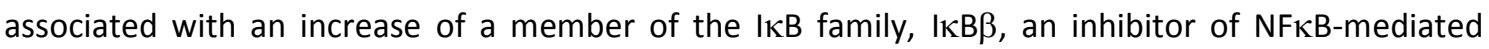
transcriptional activation Thus, one can hypothesize that increased ROR $\alpha$ expression may exert a protective role in IAT against diet-induced inflammation, as already shown in myocytes and macrophages during acute inflammation (17-20).

We further described that this increased expression of ROR $\alpha$ did not happen into the adipocytes, but was located in the SVF, probably in immune cells, in parallel with the increase of its target-gene $I_{\kappa} B \alpha$. If this is the case, the aim of further work will be to identify which cells are indeed dysfunctional in the IAT of sg/sg vs WT mice fed the WD. Mast cells could be such potential candidate because the production of TNF $\alpha$ and IL- 6 was increased dramatically in ROR $\alpha-/-$ mast cells after LPS stimulation (33). In that field, our data are in accordance with investigations showing that ROR $\alpha$ can negatively regulate cytokine production in response to an aggression by inhibiting the NF-KB pathway (17). Very recently, in vitro overexpression of ROR $\alpha$ was shown to promote macrophage M2 polarization (34). Finally, our data also suggested that $I \kappa B \beta$, another member of the I $1 \kappa B$ family, was a new target gene of ROR $\alpha$.

However, we also confirmed the dual role of ROR $\alpha$ in inflammatory processes. Indeed, ROR $\alpha$ is expressed in resting macrophages and T lymphocytes (33) and involved in the basal transcription of several cytokines such as IL6 (35), IL17 (36), PAI-1 (37) and one chemokine, CCL2 (38) in different kind 
of immune cells. Accordingly, our analysis of several cytokines expression or amount show for the first time that the basal inflammatory status of each fat depot is greatly lowered in $\mathrm{sg} / \mathrm{sg}$ mice as compared to WT mice on chow diet, and not only after HF feeding as previously shown (13). This decreased basal expression of cytokines is particularly pronounced in IAT and could be related to several missing or dysfunctional immune cells in $\mathrm{sg} / \mathrm{sg}$ fat depot $(33,36,39)$. In that field, the high insulin sensitivity of adipocytes in $\mathrm{sg} / \mathrm{sg}$ mice could be also related to their low inflammation environment, in addition to their small size.

In conclusion, the present study suggests a role for ROR $\alpha$ in participating to the healthy expansion of inguinal subcutaneous fat in mice and opens a new perspective in the mechanisms underlying the different expansion way of fat depots according to their localization.

\section{Acknowledgments}

We greatly thank Tatiana Ledent and Laetitia Dinard for their help in sg/sg mice housing facilities and Marie-Pauline Garcia for mice genotyping. We also thank the National Institute of Health for the free availability of the Image J software.

\section{References}

1. Nishimura S, Manabe I, Nagai R. Adipose tissue inflammation in obesity and metabolic syndrome. Discov Med 2009; 8: p. 55-60.

2. Xu H, Barnes GT, Yang Q, Tan G, Yang D, Chou CJ et al. Chronic inflammation in fat plays a crucial role in the development of obesity-related insulin resistance. J Clin Invest 2003; 112:1821-1830. 
3. Feuerer M, Herrero L, Cipoletta D, Naaz A., Wong J, Nayer A et al. Lean, but not obese, fat is enriched for a unique population of regulatory T cells that affect metabolic parameters. Nat Med 2009; 15:930-9.

4. Hotamisligili GS. Inflammation and metabolic disorders. Nature 2006 ; 444:860-7.

5. Porter SA, Massaro, JM, Hoffmann U, Vasan RS, O'Donnel CJ, Fox CS. Abdominal subcutaneous adipose tissue: a protective fat depot? Diabetes Care 2009; 32:1068-75.

6. Tran TT, Yamamoto Y, Gesta S, Kahn CR. Beneficial effects of subcutaneous fat transplantation on metabolism. Cell Metab 2008; 7:410-20.

7. Wang QA, Tao C, Gupta RK, Scherer PE. Tracking adipogenesis during white adipose tissue development, expansion and regeneration. Nature 2013;19:1338-44.

8. Chau YY, Bandiera R, Serrels A, Martinez-Estrada OM, Qing W, Lee M, et al. Visceral and subcutaneous fat have different origins and evidence supports a mesothelial source. Nat Cell Biol. 2014; 16:367-375.

9. Jeffery E, Wing A, Holtrup B, Sebo Z, Kaplan JL, Saavedra-Pena R et al. The adipose tissue microenvironment regulates depot-specific adipogenesis in obesity. Cell metabolism 2016; 24:142-150.

10. Pearen MA, Muscat GEO. Orphan nuclear receptors and the regulation of nutrient metabolism: understanding obesity. Physiology (Bethesda) 2012; 27: 156-66.

11. Fitzsimmons RL, Lau P, Muscat GEO. Retinoid-related orphan receptor alpha and the regulation of lipid homeostasis. J Steroid Biochem Mol Biol 2012; 130 :159-68.

12. Lau P, Fitzsimmons RL, Raichur S, Wang S-C, Lechtken A, Muscat GEO. The orphan nuclear receptor, RORalpha, regulates gene expression that controls lipid metabolism: staggerer (SG/SG) mice are resistant to diet-induced obesity. The Journal of biological chemistry 2008; 283:18411-21. 
13. Kang HS, Okamoto K, Takeda Y, Beak JY, Gerrish K, Bortner CD et al. Transcriptional profiling reveals a role for RORalpha in regulating gene expression in obesity-associated inflammation and hepatic steatosis. Physiol Genomics 2011; 43:818-28.

14. Duez H, Duhem C, Laitinen S, Patole PS, Abdelkarim M, Bois-Joyeux B, et al. Inhibition of adipocyte differentiation by RORalpha. FEBS letters 2009; 583:2031-6.

15. Ohoka N, Kato S, Takahashi Y, Hayashi H, Sato R. The orphan nuclear receptor RORalpha restrains adipocyte differentiation through a reduction of C/EBPbeta activity and perilipin gene expression. Mol Endocrinol 2009; 23:759-71.

16. Kadiri S, Monnier Ch, Ganbold M, Ledent T, Capeau J, Antoine B. The nuclear ROR alpha regulates adipose tissue glyceroneogenesis in addition to hepatic gluconeogenesis. Am J Physiol Endocrinol Metab 2015; 309:E105-E114.

17. Delerive P, Monte D, Dubois G, Trottein F, Fruchart-Najib J, Mariani J et al., The orphan nuclear receptor ROR alpha is a negative regulator of the inflammatory response. EMBO Rep $2001 ; 2: 42-8$.

18. Kopmels B, Mariani J, Delhaye-Bouchaud N, Audibert F, Fradelezi D, Wollman EE. Evidence for a hyperexcitability state of staggerer mutant mice macrophages. J Neurochem 1992;58: 192-9.

19. Kopmels B, Wollman EE, Guastavino JM, Delhaye-Bouchaud N, Fradelezi D, Mariani J. Interleukin-1 hyperproduction by in vitro activated peripheral macrophages from cerebellar mutant mice. J Neurochem 1990; 55:1980-5.

20. Garcia JA, Volt H, Venegas C, Doerrier C, Escames G, Lopez LC, Acuna-Castroviejo D. Disruption of the NFKB/NLRP3 connection by melatonin requires retinoid receptor-related orphan recepto $\alpha$ and blocks the septic response in mice. FASEB J 2016; 29:3863-3875.

21. Lau P, Fitzsimmons RL, Pearen MA, Watt MJ, Muscat GEO. Homozygous staggerer (sg/sg) mice display improved insulin sensitivity and enhanced glucose uptake in skeletal muscle. Diabetologia 2011; 54: 1169-80. 
22. Dusaulcy R, Rancoule C, Grès S, Wanecq E, Colom A, Guigné C, et al. Adipose-specific disruption of autotaxin enhances nutritional fattening and reduces plasma lysophosphatidic acid. J Lipid Res 2011;52: 1247-1255.

23 Lau P, Tuong ZK, Wang SC, Fitzsimmons RL, Goode JM, Thomas, GP et al. ROR $\alpha$ deficiency and decreased adiposity are associated with induction of thermogenic gene expression in subcutaneous white adipose and brown adipose tissue. Am J Physiol Endocrinol Metab 2015; 308:E159-E171.

24. Kosteli A, Sugaru E, Haemmerle G, Martin JF, Lei J, Zechner R, Ferrante AW Jr. Weight loss and lipolysis promote a dynamic immune response in murine adipose tissue. J Clin Invest 2010; 120: 3466-79.

25. Altintas MM, Azad A, Nayer B, Contreras G, Zaias J, Faul C et al. Mast cells, macrophages, and crown-like structures distinguish subcutaneous from visceral fat in mice. J Lipid Res 2011; 52:480-8.

26. Strissel KJ, Stancheva Z, Miyoshi H, Perfield JW $2^{\text {nd }}$, DeFuria J, Jick Z et al. Adipocyte death, adipose tissue remodeling, and obesity complications. Diabetes 2007;56:2910-8.

27. Villena JA, Cousin B, Pénicaud L, Casteilla L. Adipose tissues display differential phagocytic and microbicidal activities depending on their localization. Int J Obes Relat Metab Disord 2001; 25: 1275-80.

28. Du ZY, Ma T, Lock EJ, Hao Q, Kristiansen K, Froyland L, Madsen L. Depot-dependent effects of adipose tissue explants on co-cultured hepatocytes. PLoS One 2011; 6: e20917.

29. Tsai YS, Tsai PJ, Jiang MJ, Chou TY, Pendse A, Kim HS, Maeda N. Decreased PPAR gamma expression compromises perigonadal specific fat deposition and insulin sensitivity. $\mathrm{Mol}$ Endocrinol $2009 ; 23: 1787-1798$.

30. Macotela Y, Emanuelli B, Mori A, Gesta S, Schuz TJ, Tseng Y-H, Kahn C.R. Intrinsic differences in adipocyte precursor cells from different white fat depots. Diabetes 2012; 61:1691-1699. 
31. Galli SJ, Grimbaldeston M, Tsai M. Immunomodulatory mast cells: negative, as well as positive, regulators of immunity. Nat Rev Immunol 2008;8:478-486

32. Weidner N, Austen KF. Evidence for morphologic diversity of human mast cells. An ultrastructural study of mast cells from multiple body sites. Lab. Invest. 1990; 63: 63 - 72

33. Dzhagalov I, Giguère V, He Y-W. Lymphocyte development and function in the absence of retinoid acid receptor-related orphan receptor $\alpha$. J Immunol 2004; 173: 2952-2959.

34. Xiao L, Zhang Z, Luo X, Yang H, Li F, Wang N. Retinoid acid receptor-related orphan receptor alpha $(\mathrm{ROR} \alpha)$ regulates macrophage M2 polarization via activation of AMPK $\alpha$. Molecular Immunol 2016; 80:17-23

35. Journiac N, Jolly S, Jarvis C, Gautheron V, Rogard M, Trembleau A. et al. The nuclear receptor ROR(alpha) exerts a bi-directional regulation of IL-6 in resting and reactive astrocytes. Proc Natl Acad Sci USA 2009; 106: 21365-70.

36. Yang XO, Pappu BP, Nurieva R, Akimzhanov A, Kang HS, Chung Y et al. T helper 17 lineage differentiation is programmed by orphan nuclear receptors ROR $\alpha$ and RORY. Immunity 2008;28: 29-39.

37. Wang J, Yin L, Lazar MA. The orphan nuclear receptor Rev-erba regulates circadian expression of plasminogen activator inhibitor type 1. J Biol Chem 2006;281:33842-33848

38. Sato S, Sakurai T, Ogasawara J, Takahashi M, Izawa T, Imaizumi K, Taniguchi N, Ohno H, Kizaki T. A circadian clock gene, Rev-erb $\alpha$, regulates the inflammatory function of macrophages through the negative regulation of ccl2 expression. J. Immunol 2014; 192 :407417.

39. Halim TY, MacLaren A, Romanish MT, Gold MJ, Mc Nagny KM, Takei F. Retinoic- acidreceptor-related orphan nuclear receptor $\alpha$ is required for natural helper cell development and allergic inflammation. Immunity 2012; 37: 463-474. 


\section{Figure legends}

Figure 1: The inflammatory profiles of epididymal and inguinal fat depots differ according to the genotype and in response to WD

Expression of mRNAs for various inflammatory markers in the EAT (A) and IAT (B) of WT mice and sg/sg mice fed the SD and the WD. Relative mRNA amounts were calculated vs HPRT mRNA. The data are normalized to the mRNA levels measured in the respective fat depots of WT mice fed the SD. The insert $\left(A^{\prime}\right.$ and $\left.B^{\prime}\right)$ shows the WD-effect according to the genotype. All data are presented as means +/-SD ( $n=6$ to 12 per group). ${ }^{*} p<0.05,{ }^{*} p<0.01$ compared to SD-fed WT mice and \# $p<0.05, \# \#$ $p<0.01$ compared to SD-fed mice of the same genotype.

Figure 2: Proportion of CD68-positive macrophages and cytokine secretion according to the genotype and in response to WD

(A) CD68-positive macrophages in EAT and IAT as determined by immunohistochemistry and calculated as a percentage of the total number of adipocytes ( $n=4$ mice per group).

(B) CCL2 secretion by EAT and IAT explants. (C) TNF $\alpha$ and IL10 secretion by IAT. ( $\mathrm{n}=5$ mice per group). All data are presented as means $+/-S D . * * * p<0.005$ and $* p<0.05$ compared to SD-fed WT mice; \#\# $p<0.01, \# \# \#$ p $<0.005$ compared to SD-fed sg/sg mice.

Figure 3: The influence of diet on the insulin sensitivities of EAT and IAT in WT and sg/sg mice.

The insulin sensitivity of the two fat depots was measured as phosphorylation of Akt after incubation of explants with $100 \mathrm{nM}$ insulin for $10 \mathrm{mn}$. Increased PAkt to Akt ratio indicates greater insulin sensitivity.

(A, B) Comparison of inguinal and epididymal fat depots in WT and sg/sg mice fed the SD.

(A) representative western blot of $\mathrm{P}-\mathrm{Akt}$ and $\mathrm{Akt}$ bands and $(\mathrm{B})$ relative 2-DOG incorporation in response to insulin. $* p<0.05, * * p<0.01$ compared to IAT of SD-fed WT mice.

(C, D) Effect of WD on epididymal or inguinal fat depots in WT and sg/sg mice. 
Representative western blots are shown on the left and quantification of the PAkt and Akt bands is plotted on the right $(n=6) .{ }^{*} p<0.05, * * p<0.01$ compared to SD-fed WT mice and \# $\mathrm{p}<0.05$ compared to SD-fed sg/sg mice.

Figure 4: ROR $\alpha$ increased in IAT of WT mice fed the WD in a depot-specific manner

A) Amounts of ROR $\alpha$ mRNA, normalized to the amount of $18 \mathrm{~S}$ rRNA in each sample, and expressed relative to the level of expression in the EAT of WT mice $(n=6) .{ }^{* *} p<0.01$ compared to SD-fed EAT of WT mice

B) Circadian variation of ROR $\alpha$ protein in IAT and EAT of WT mice fed the SD. Mice were euthanazied every three hours after the beginning of the fasting period (ZT=Zeitgeber time, ZTO is the start of the light phase, ZT12 is the beginning of the dark phase during a 24-hour light-dark cycle).

C) Western blot of ROR $\alpha$ protein amount in IAT of WT mice fed the WD vs the SD.

D) Left, representative western blot of IКB $\beta$ protein amount in IAT and EAT from WT mice fed the WD vs the SD; right, quantification of the $1 \kappa B \beta$ protein amount vs tubulin $(n=4) . *^{*} p<0.05$ compared to SD-fed WT mice.

Figure 5: ROR $\alpha$ and IKB $\alpha$ increased in the stroma vascular fraction (SVF) of WT mice fed the WD.

A) Representative western blots and quantification of $\operatorname{ROR} \alpha$ vs tubulin amount $(n=4)$. The purity of each fraction was verified by the level of F4/80 and adiponectin expression used as marker of macrophages and adipocytes respectively.

B) Representative western blot and quantification of $\mathrm{I} \kappa \mathrm{B} \alpha$ protein amount in SVF of IAT and EAT from WT mice fed the WD vs the SD $(n=4)$. All data are presented as means $+/-\mathrm{SD} .{ }^{*} p<0.05$, ${ }^{* *} \mathrm{p}<0.01$ compared to respective cells fraction of SD-fed WT mice. 
Fig 1 R1 Kadiri
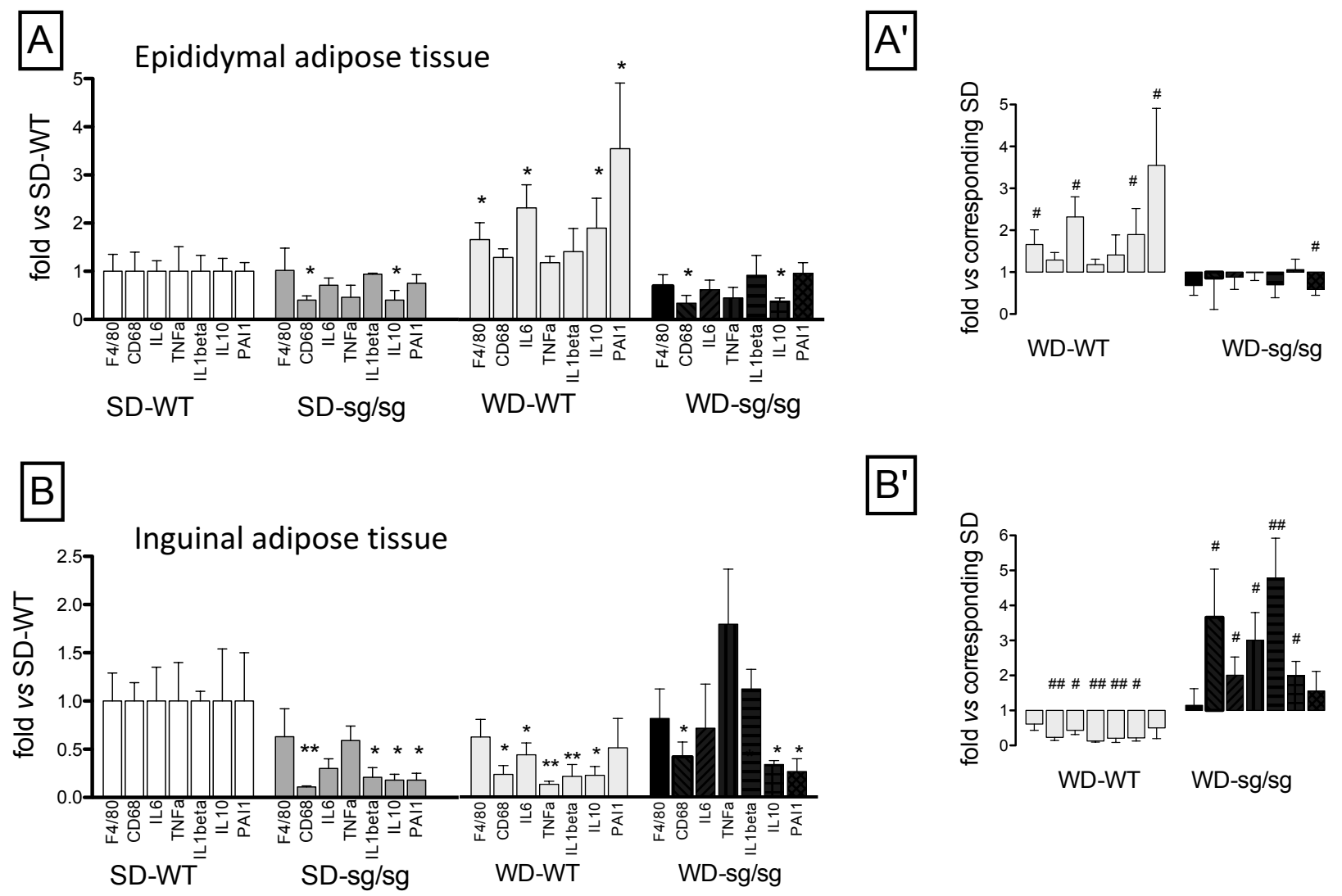

$\mathrm{B}^{\prime}$

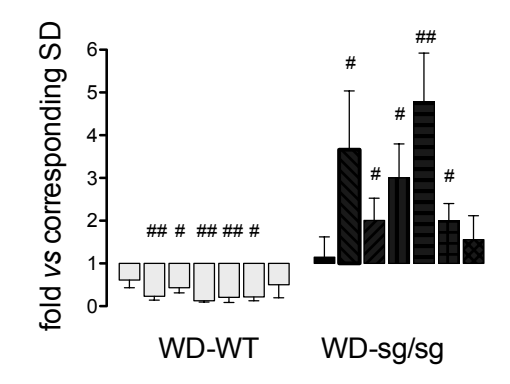




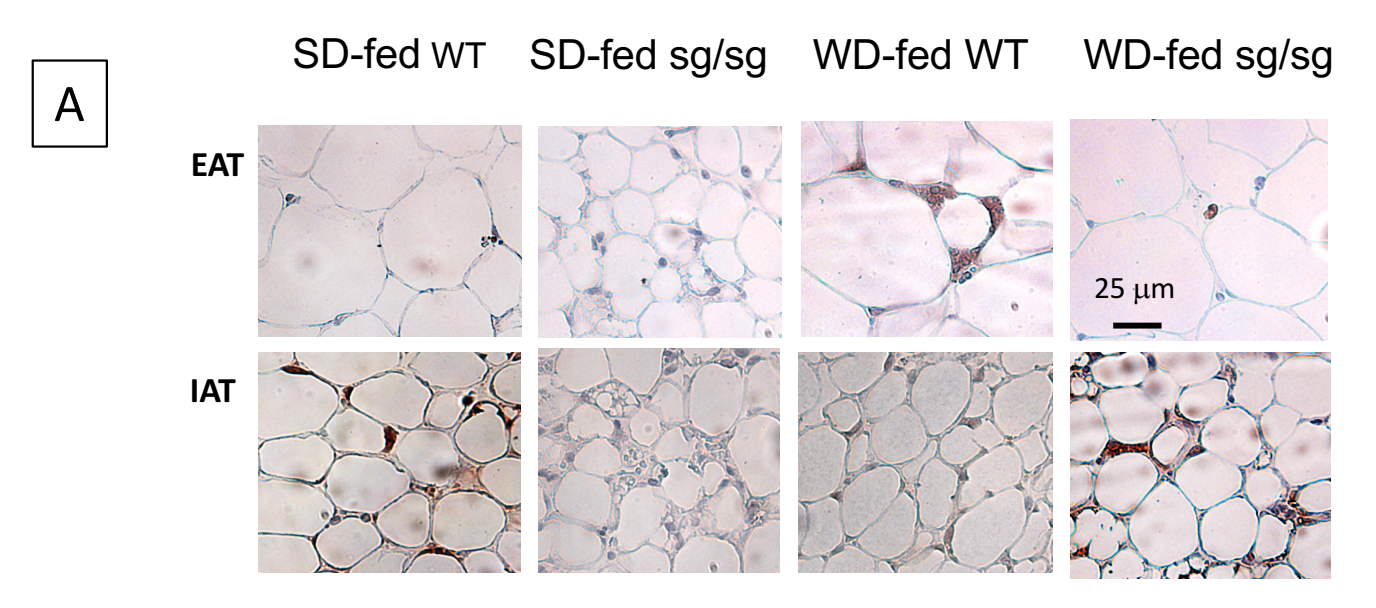

C
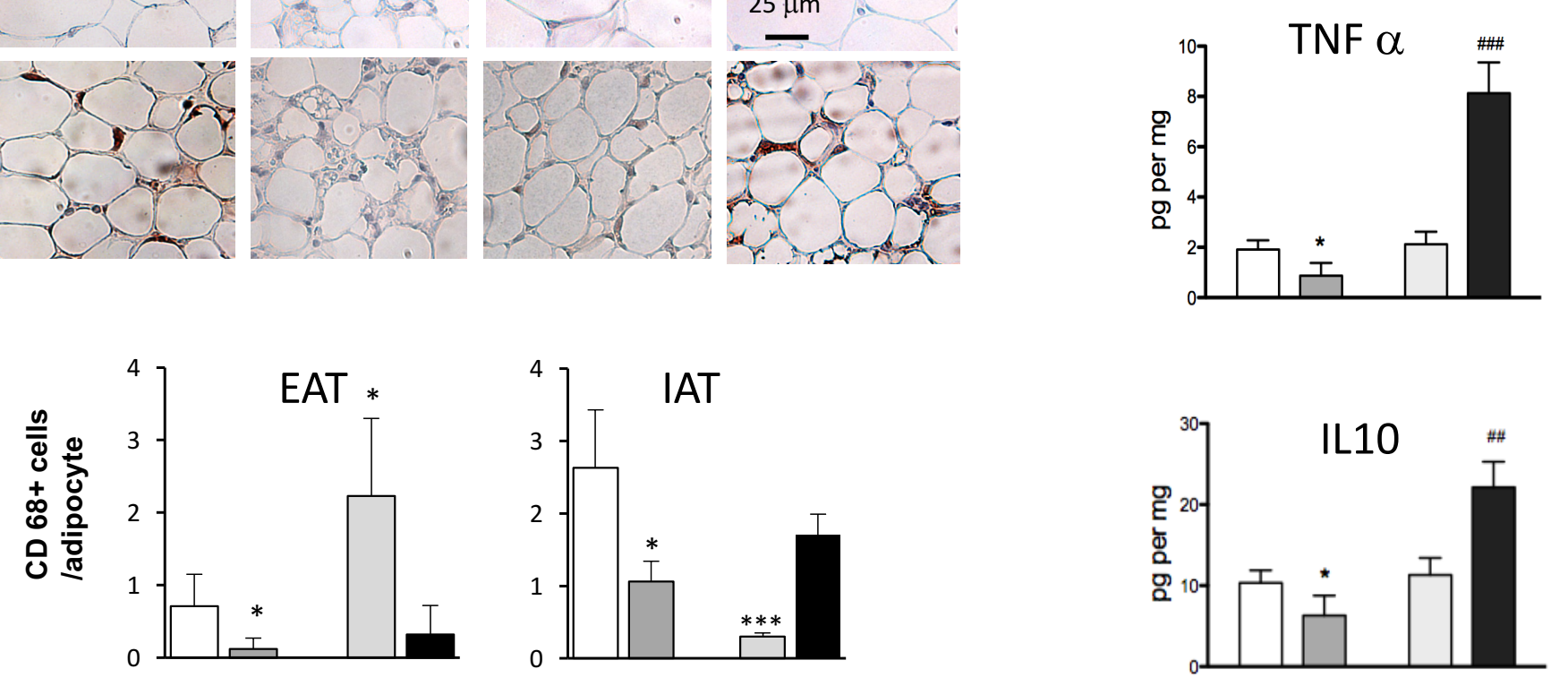

B
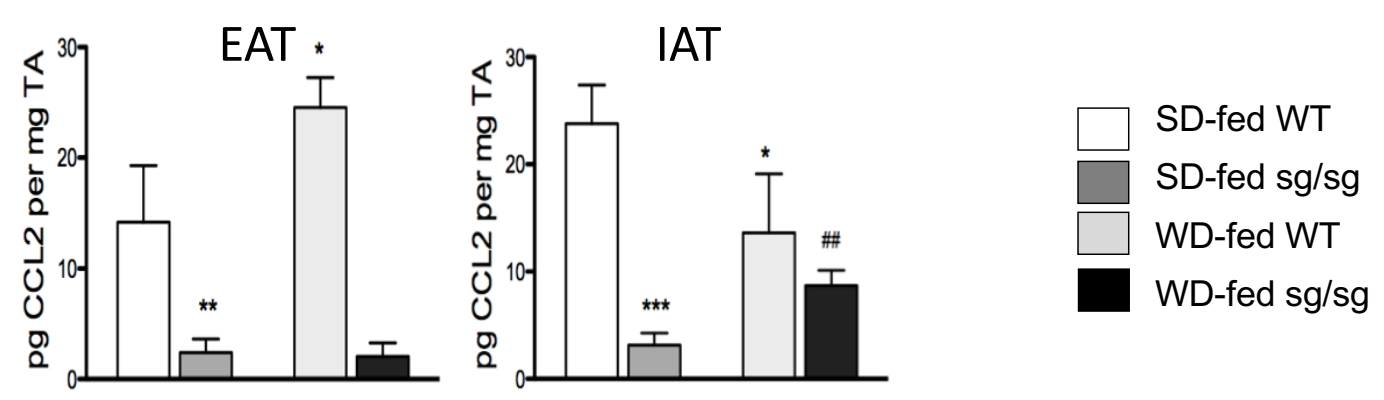


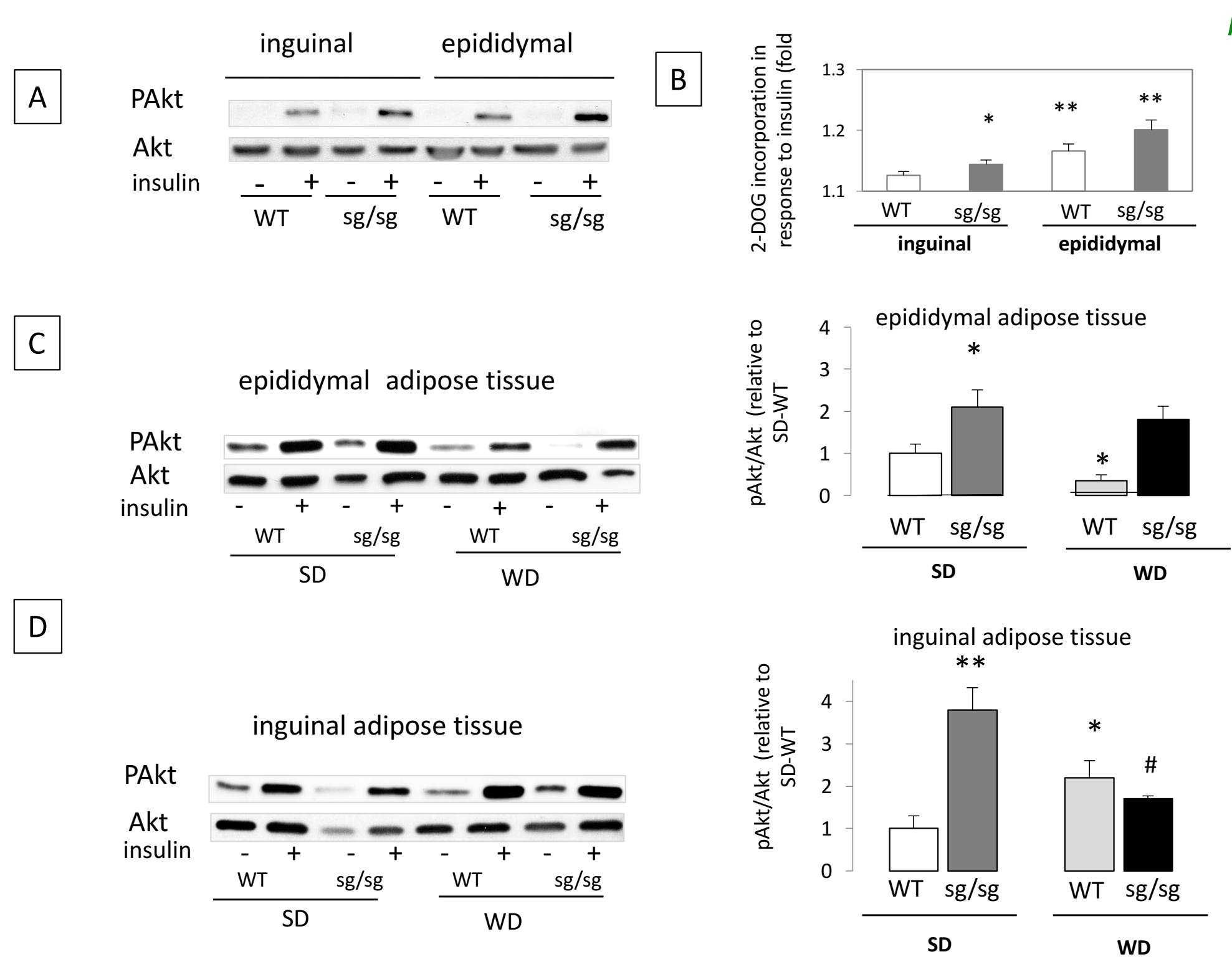

Fig 3 R1 Kadiri 

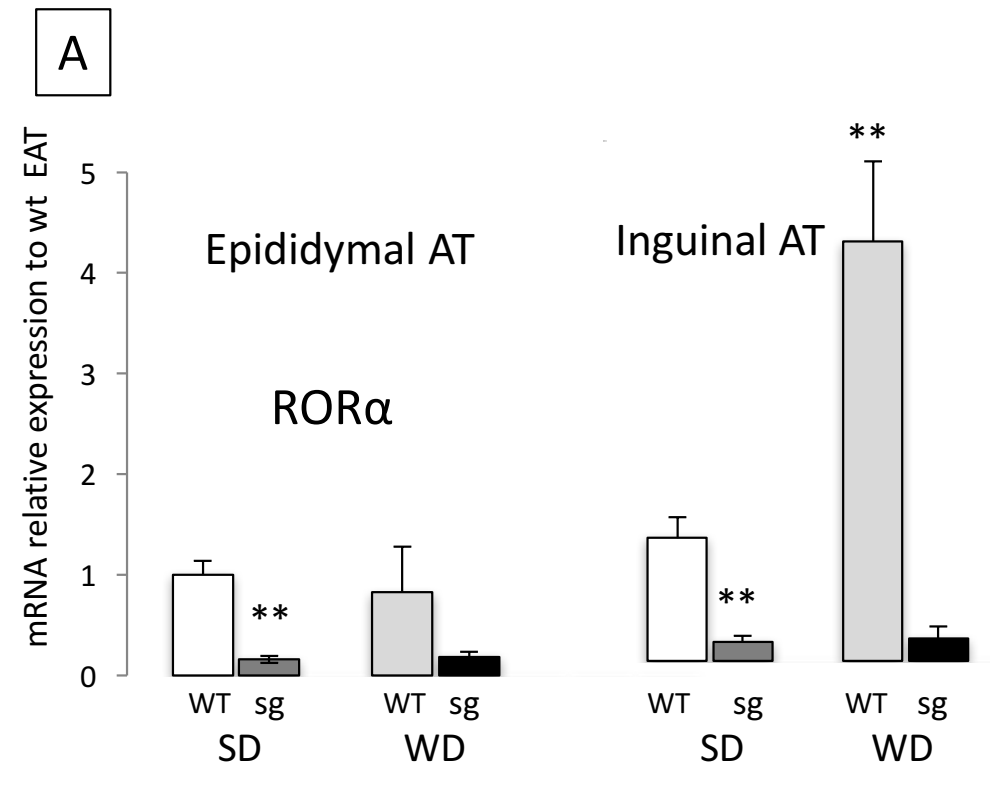

B

Fig 4 R1 Kadiri

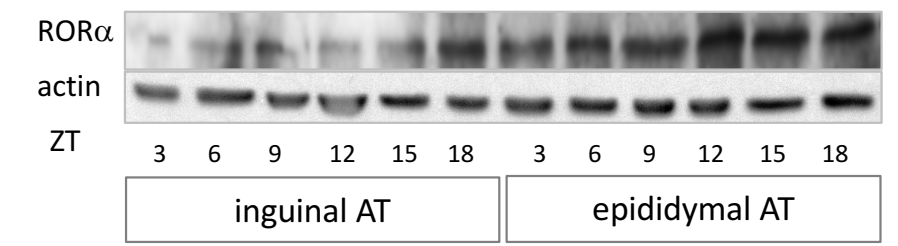

SD-fed WT

SD-fed sg/sg

WD-fed WT

WD-fed sg/sg

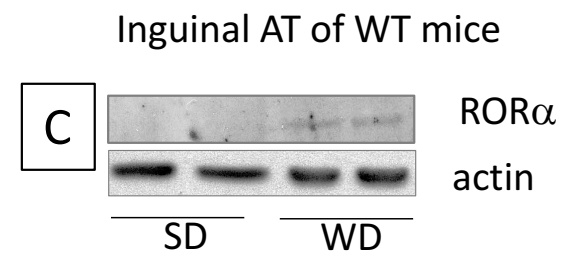

D
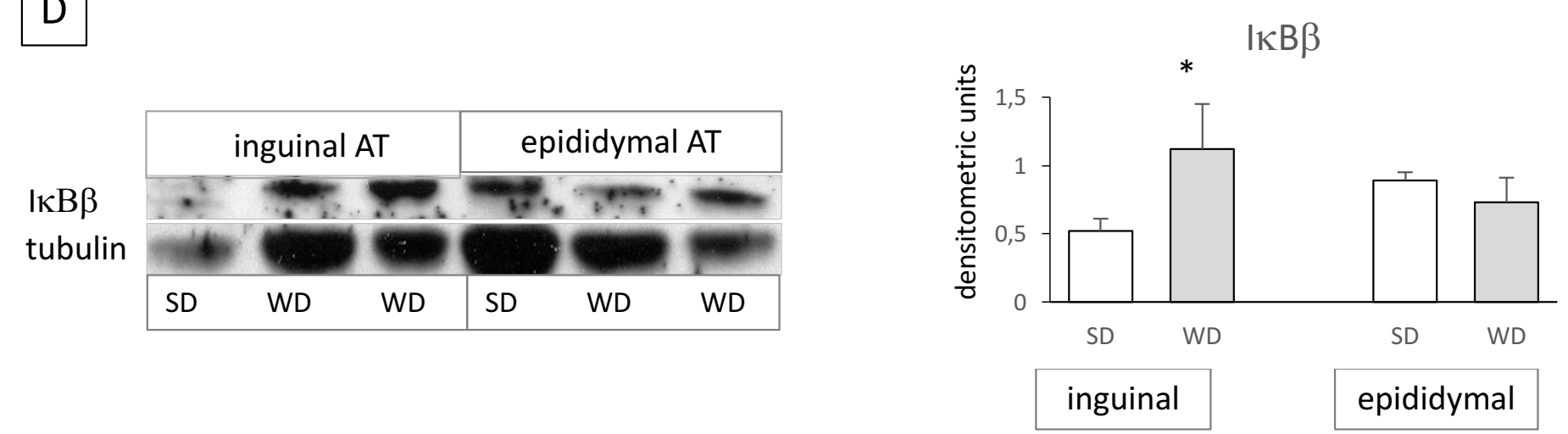
A
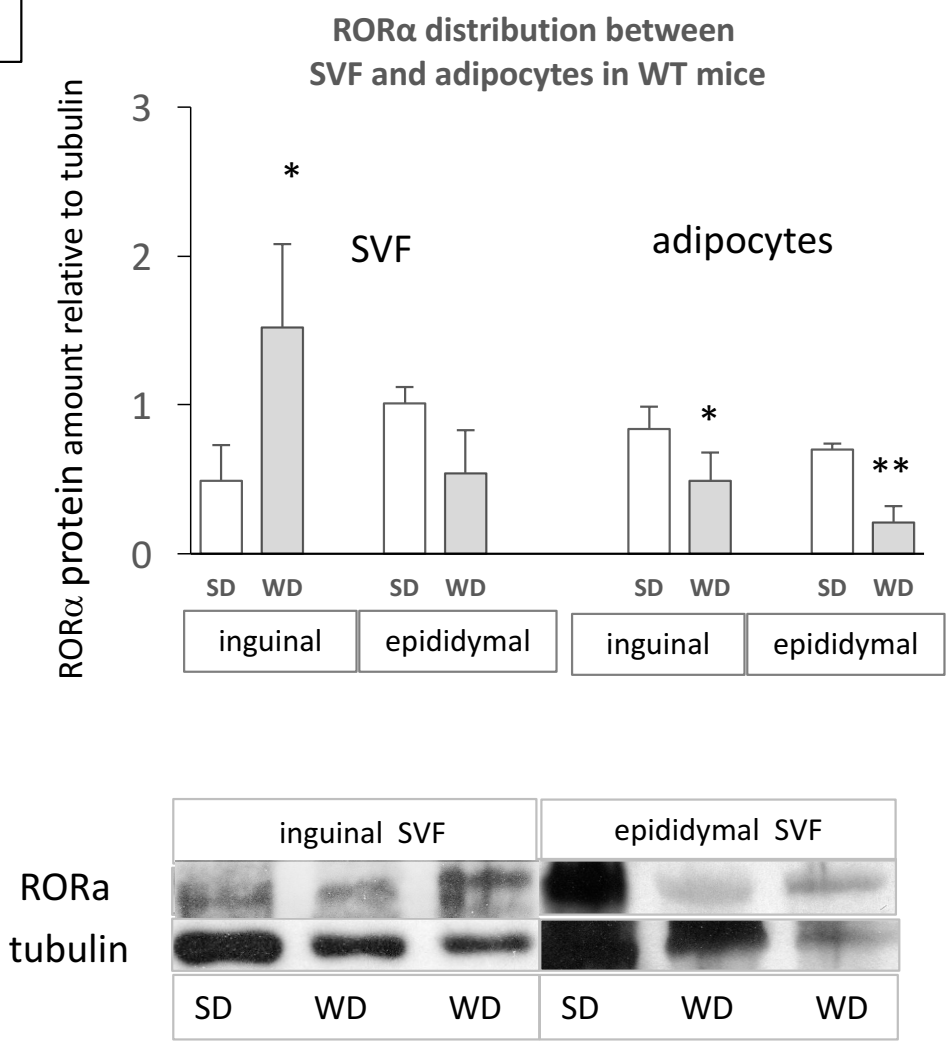

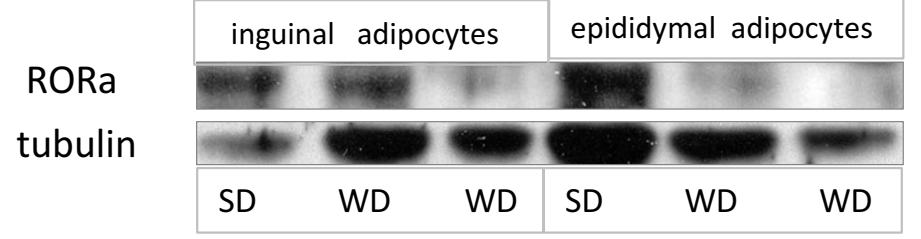

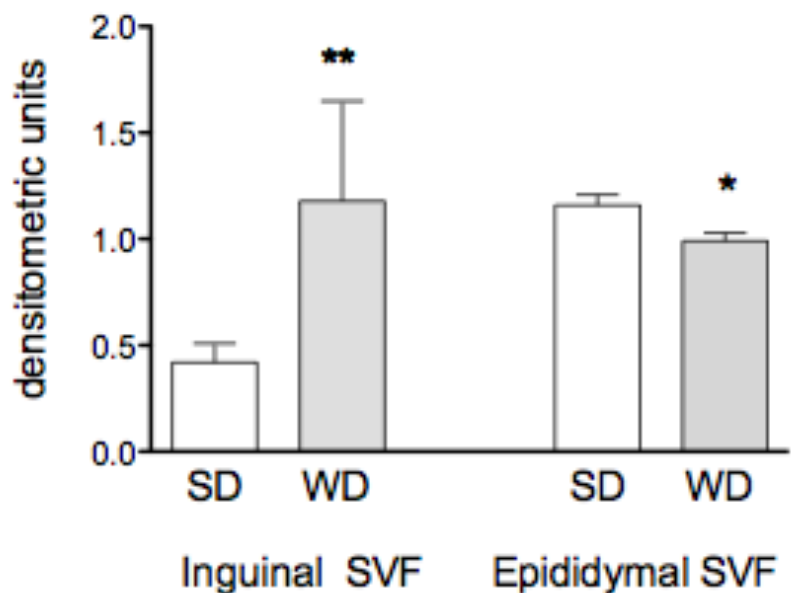

inguinal SVF

epididymal SVF

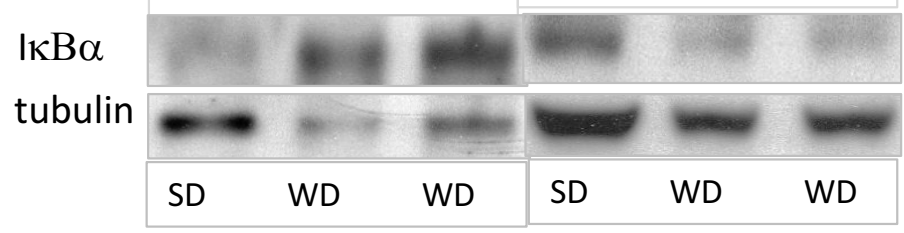




\section{Depot-specific response of adipose tissue to diet-induced inflammation: the retinoid-related orphan receptor $\alpha$ involved?}

Sarah Kadiri ${ }^{1,2}$, Martine Auclair $^{1,2}$, Jacqueline Capeau ${ }^{1,2}$ and Bénédicte Antoine ${ }^{1,2,3}$

1 Inserm U938, Paris F75012, France

2 UPMC Université Paris 06, UMR_S 938, Paris, F-75012, France

3 CNRS, UMR_S 938, Paris, F-75012, France

Corresponding author: B. Antoine

Address for correspondence:

Faculté de Médecine site Saint-Antoine, 27 Rue Chaligny, 75012 Paris, France

tel 331400113 51, fax 331400114 32,

benedicte.antoine@inserm.fr

Running title: ROR $\alpha$ involved in fat depot-specific inflammation

Keywords: Fat depots, inflammation, ROR $\alpha$, western diet, insulin sensitivity

Word Count: 3499

Funding: SK was the recipient of $\mathrm{PhD}$ grants from the Ministère de l'Education Nationale et de la Recherche and from CODDIM. BA is a CNRS researcher. 


\section{Legends of supplementary figures}

\section{Figure S1: Figure S1: Fat parameters in WT and sg/sg mice fed SD or WD}

A. Relative tissue weights of total white adipose tissue and various fat depots and of liver in WT and $\mathrm{sg} / \mathrm{sg}$ mice.

B. Histology of epididymal and inguinal adipose tissue (EAT and IAT). Mean adipocyte size and proportion of pre-adipocytes vs adipocytes. Sections of adipose tissue were stained with hematoxylin and eosin and the area of adipocytes in each section was calculated by using Image J software (Sun Microsystems). The pre-adipocytes (Pref-1 positive cells =red cells) were determined by immunohistochemistry and quantified as a percentage of the total number of adipocytes (blue nucleus) ( $n=4$ mice per group). All data are presented as means $+/-S D .{ }^{*} p<0.05$ compared to SD-fed WT mice and \# $p<0.05$ compared to SD-fed sg/sg mice

Figure S2: Compared inflammation status of IAT vs EAT according to the genotype and the diet. Expression of mRNAs for various inflammatory markers in the IAT by comparison with respective EAT of WT mice and sg/sg mice fed the SD (A) and fed the WD (B). This is an alternative representation of the data shown by Fig 1, except normalized to $18 \mathrm{~S}$ rRNA. * $\mathrm{p}<0.05$ IAT compared to EAT. 
Fig S1, Kadiriet al
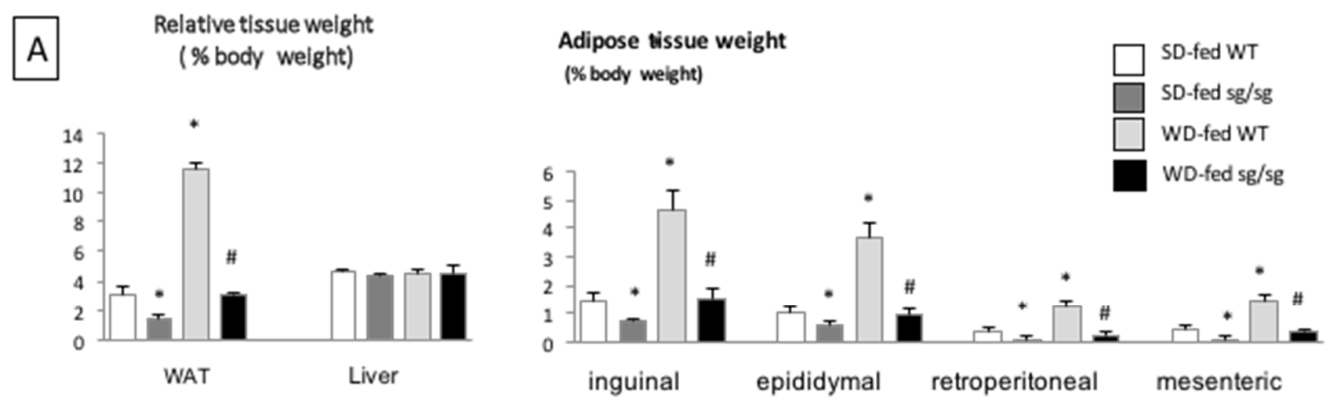

B SD-fed WT SD-fed sg/sg WD-fed WT WD-fed sg/sg

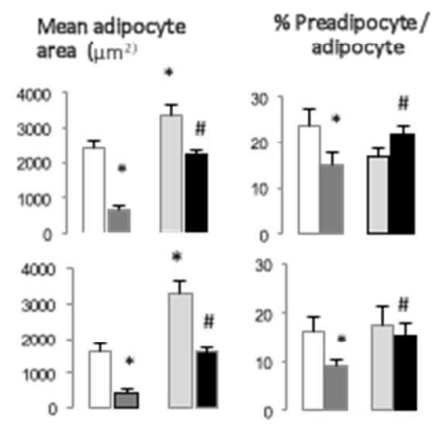

EAT

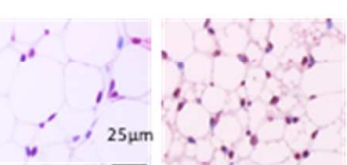

IAT
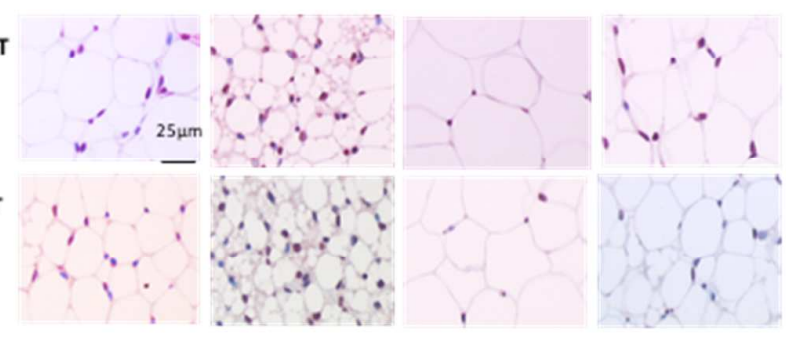
Fig S2, Kadiri et al
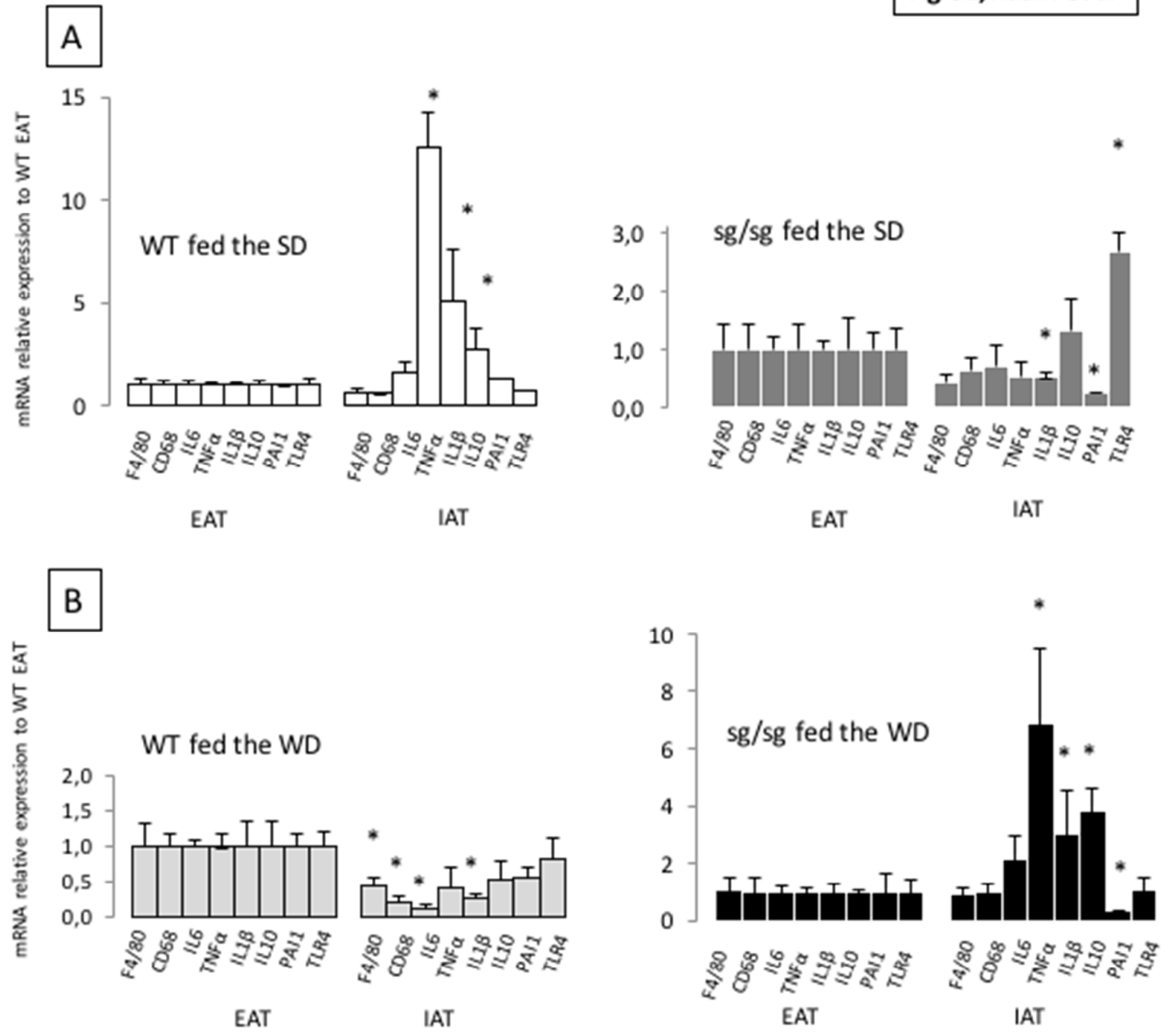